ARTICLE

Received 16 Jun 2014 | Accepted 12 Dec 2014 | Published 28 Jan $2015 \quad$ DOl: 10.1038/ncomms7128

\title{
Seeded growth of highly crystalline molybdenum disulphide monolayers at controlled locations
}

\author{
Gang Hee Han ${ }^{1,2}$, Nicholas J. Kybert ${ }^{1}$, Carl H. Naylor ${ }^{1}$, Bum Su Lee ${ }^{3}$, Jinglei Ping ${ }^{1}$, Joo Hee Park ${ }^{3}$, \\ Jisoo Kang 4 , Si Young Lee ${ }^{2}$, Young Hee Lee ${ }^{2}$, Ritesh Agarwal ${ }^{3} \&$ A.T. Charlie Johnson,3,4
}

Monolayer transition metal dichalcogenides are materials with an atomic structure complementary to graphene but diverse properties, including direct energy bandgaps, which makes them intriguing candidates for optoelectronic devices. Various approaches have been demonstrated for the growth of molybdenum disulphide $\left(\mathrm{MoS}_{2}\right)$ on insulating substrates, but to date, growth of isolated crystalline flakes has been demonstrated at random locations only. Here we use patterned seeds of molybdenum source material to grow flakes of $\mathrm{MoS}_{2}$ at predetermined locations with micrometre-scale resolution. $\mathrm{MoS}_{2}$ flakes are predominantly monolayers with high material quality, as confirmed by atomic force microscopy, transmission electron microscopy and Raman and photoluminescence spectroscopy. As the monolayer flakes are isolated at predetermined locations, transistor fabrication requires only a single lithographic step. Device measurements exhibit carrier mobility and on/off ratio that exceed $10 \mathrm{~cm}^{2} \mathrm{~V}^{-1} \mathrm{~s}^{-1}$ and $10^{6}$, respectively. The technique provides a path for in-depth physical analysis of monolayer $\mathrm{MoS}_{2}$ and fabrication of $\mathrm{MoS}_{2}$-based integrated circuits.

\footnotetext{
${ }^{1}$ Department of Physics and Astronomy, University of Pennsylvania, 2095 33rd Street, Philadelphia, Pennsylvania 19104 6396, USA. ${ }^{2}$ Center for Integrated Nanostructure Physics (CINAP), Institute for Basic Science (IBS), Sungkyunkwan University, Suwon 440 746, South Korea. ${ }^{3}$ Department of Materials Science and Engineering, University of Pennsylvania, Philadelphia, Pennsylvania 19104 6272, USA. ${ }^{4}$ Nano/Bio Interface Center, University of Pennsylvania, Philadelphia, Pennsylvania 19104 6272, USA. Correspondence and requests for materials should be addressed to G.H.H. (email: ganghee@sas.upenn.edu) or to A.T.C.J. (email: cjohnson@physics.upenn.edu).
} 
T he presence of an intrinsic direct energy bandgap in molybdenum disulphide $\left(\mathrm{MoS}_{2}\right)$ monolayers makes this two-dimensional material of both fundamental and technological interest ${ }^{1-10}$. Several synthetic methods have been reported, including sulphiding of Mo thin film ${ }^{11}$, and annealing of ammonium tetrathiomolybdate, which has both Mo and S components ${ }^{12}$. One promising technique is the growth of $\mathrm{MoS}_{2}$ flakes by chemical vapour deposition (CVD) of molybdenum trioxide $\left(\mathrm{MoO}_{3}\right)$ and solid sulphur, which can yield highly crystalline, predominantly monolayer material ${ }^{13-13}$. However, this method typically leads to growth of $\mathrm{MoS}_{2}$ flakes in uncontrolled locations on the substrate, which limits its utility for many applications. Control over the position of $\mathrm{MoS}_{2}$ flakes on oxidized silicon has been reported through the use of stamping $^{18}$ and masking ${ }^{19}$ techniques, but neither of these allowed for the creation of large (tens of micrometres) monolayer domains at predetermined locations with micrometre-scale resolution. Substrate patterning has been used to control nucleation and growth of crystalline flakes of two-dimensional topological insulator materials on mica ${ }^{20}$, but it remains unclear whether this approach can be generalized to $\mathrm{MoS}_{2}$ on oxidized silicon. Seeding techniques that allow growth at defined locations are well established for graphene ${ }^{21,22}$, and equivalent approaches for $\mathrm{MoS}_{2}$ synthesis are highly desirable.

Here we report the use of lithographically patterned islands of $\mathrm{MoO}_{3}$ or ammonium heptamolybdate (AHM) as seed material for the growth of crystalline $\mathrm{MoS}_{2}$ monolayers at predefined locations on oxidized silicon substrates. $\mathrm{MoS}_{2}$ grown by this approach shows many favourable characteristics, for example, high crystallinity; excellent optical properties, including a direct bandgap, as indicated by photoluminescence (PL) and reflection measurements; and carrier mobility ca $10 \mathrm{~cm}^{2} \mathrm{~V}^{-1} \mathrm{~s}^{-1}$. Because the method enables control over the location of discrete $\mathrm{MoS}_{2}$ flakes, integration into device structures is straightforward using optical lithography, with no need for an etching step. Although some applications may require further study to achieve control over the precise shape and size of the $\mathrm{MoS}_{2}$ flakes, the methodology presented here opens a path towards scalable production of high-quality devices and circuits based on $\mathrm{MoS}_{2}$ and other transition metal dichalcogenides.

\section{Results}

Growth process. Figure 1 is a schematic of the $\mathrm{MoS}_{2}$ growth process (see the Methods section and Supplementary Fig. 1 for details). The process begins with patterning of an array of square windows, typically $5-10 \mu \mathrm{m}$ on a side, by e-beam or optical lithography. Next, molybdenum-containing seed material is deposited into the windows, either by thermal evaporation of $\mathrm{MoO}_{3}$ or by spin-casting of a saturated solution of AHM in water. The AHM solution collects in the square wells but there is almost no deposition of AHM on the resist layer, which is not wet by the solution. Spin coating of AHM has advantages of speed, simplicity and requiring no equipment beyond a standard spin coater. The resist and unwanted molybdenum source material are removed in a standard lift-off step.

At this point, the molybdenum-containing seed particle has a shape that depends upon the relative humidity and other details of the deposition process. To improve the uniformity of the seed shape and subsequent $\mathrm{MoS}_{2}$ growth, an aggregation step is performed (see Supplementary Figs 2 and 3) where the sample temperature is reduced to below the dew point using a Peltier cooler. Owing to the hygroscopic nature of $\mathrm{MoO}_{3}$ and $\mathrm{AHM}$, water collects on the surface of the seed, which is transformed into a spherical-cap shape. This reduces the surface area of the seed particles and makes them more uniform, leading to more efficient and reproducible $\mathrm{MoS}_{2}$ growth. This step ensures that the seed particle is sufficiently thick to not be consumed by evaporation during the heat-up phase of the growth process. This step is essential when AHM is used as the moblydenum source, as it is quite thin when initially deposited $(\sim 50 \mathrm{~nm}$, see Supplementary Fig. 2). In contrast, the aggregation step improves the growth reproducibility but is not required when evaporation is used to deposit a $\mathrm{MoO}_{3}$ seed of thickness approximately $150 \mathrm{~nm}$ or greater. The aggregation step is followed by an annealing step at $300{ }^{\circ} \mathrm{C}$ in a nitrogen atmosphere, which converts AHM to $\mathrm{MoO}_{3}$ (refs 23,24).

The next step in the process is chemical treatment of the $\mathrm{SiO}_{2}$ surface, which has been shown to facilitate growth of $\mathrm{MoS}_{2}$. For example, it was reported that $\mathrm{MoS}_{2}$ growth was promoted when the $\mathrm{SiO}_{2}$ surface was coated with materials that contain carbon ring structures that persist at the $\mathrm{MoS}_{2}$ growth temperature, for example, perylene-3,4,9,10-tetracarboxylicdianhydride, perylene3,4,9,10-tetracarboxylic acid tetrapotassium salt ${ }^{13}$ or reduced graphene oxide ${ }^{13,16}$. These materials promote monolayer growth by increasing the surface adhesive energy relative to the adatom cohesive energy ${ }^{17}$. We achieved excellent results by first preparing the surface with an oxygen plasma treatment and then spincasting onto the substrate an aqueous solution of $0.1-1 \%$ sodium cholate, which contains multiple carbon ring structures.

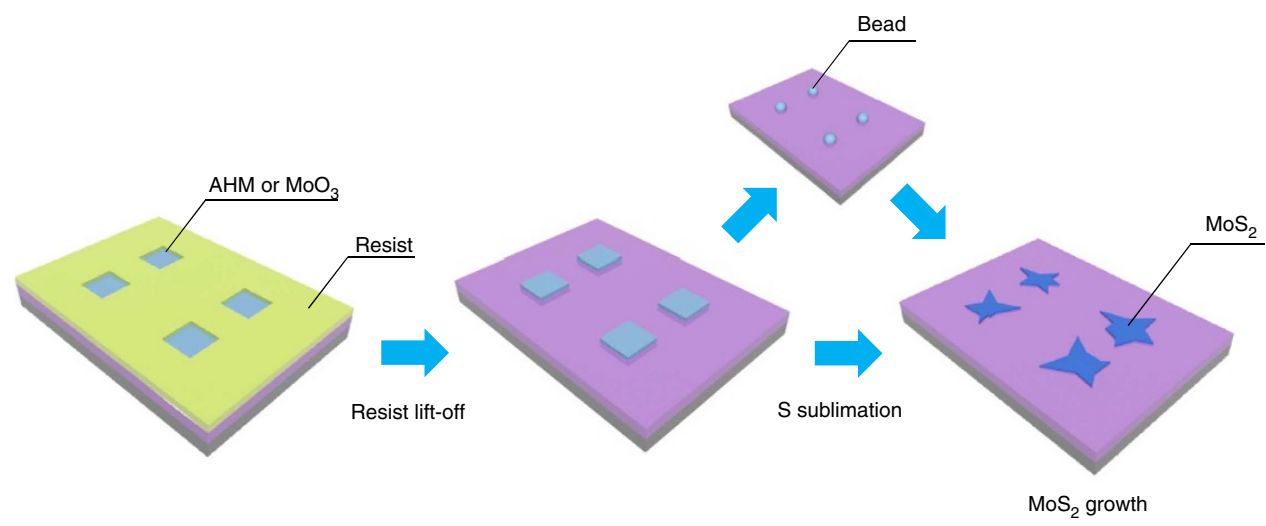

Figure 1 | Schematic of the growth process for molybdenum disulphide using patterned molybdenum sources. An array of square wells is patterned in a conventional lithographic resist. The wells are filled by spin coating with ammonium heptamolybdate $\left(\left(\mathrm{NH}_{4}\right)_{6} \mathrm{Mo}_{7} \mathrm{O}_{24} \cdot 4 \mathrm{H}_{2} \mathrm{O}, \mathrm{AHM}\right)$ solution or thermal evaporation of molybdenum trioxide $\left(\mathrm{MoO}_{3}\right)$. The resist is removed in a lift-off process. Water droplets are condensed onto the $\mathrm{AMH}$ or $\mathrm{MoO}_{3}$ by reducing the sample temperature to below the dew point using a Peltier cooler. The $\mathrm{AMH}$ or $\mathrm{MoO}_{3}$ is solubilized and forms spherical beads upon drying. $\mathrm{MoS}_{2}$ flakes are then synthesized by sublimation of solid $\mathrm{S}$ in a nitrogen environment in a tube furnace. 
For $\mathrm{MoS}_{2}$ growth, the sample was placed in the middle of the furnace, and $20-50 \mathrm{mg}$ of solid sulphur was placed at the upstream end. The entire growth process, including temperature ramp, growth and cooling, was conducted under a nitrogen flow of 700 s.c.c.m. The use of patterned molybdenum source material resulted in the growth of an array of triangular/spike-shaped flakes of monolayer $\mathrm{MoS}_{2}$ (Figs 2a and 3a,b,d). The flakes comprised multiple crystalline domains of $\mathrm{MoS}_{2}$, as analysed below (see Fig. 4). The majority of the flake region consisted of monolayer $\mathrm{MoS}_{2}$, with small regions of multilayer $\mathrm{MoS}_{2}$ and/or residual seed material observed at the centre of the flake (Fig. $3 \mathrm{~d}$ ). It was found that the formation of multilayer regions could be suppressed by optimizing the dimensions of the growth seed (see Supplementary Fig. 4). The structure is consistent with a model where growth is initiated at multiple points on the $\mathrm{MoO}_{3}$ seed to produce $\mathrm{MoS}_{2}$ crystallites that eventually merge into a larger, faceted structure. For additional data in support of this picture, see Supplementary Fig. 5. Although the sizes and locations of the flakes were primarily determined by the array of the growth seeds, it was consistently observed that flakes that grew at the edge of the array were markedly smaller than those that grew in the middle. This is presumed to reflect diffusion of source material as the furnace temperature was increased that led to shrinkage of seed particles on the edge of the array. Particles in the centre of the array are more replenished than those at the edges because of the larger number of neighbouring particles. Once sulphur sublimation and transport into the furnace occurs, the larger source particles at the centre of the array grow larger $\mathrm{MoS}_{2}$ flakes than the smaller source particles at the edges. In some experiments, small, triangular, presumably single-crystal $\mathrm{MoS}_{2}$ flakes were observed to grow in the regions between the $\mathrm{MoO}_{3}$ seeds (data not shown). This occurred during growths that were at higher temperature compared with the samples shown in Figs $2 \mathrm{a}$ and $3 \mathrm{a}$, and where sulphur was introduced at a later time.
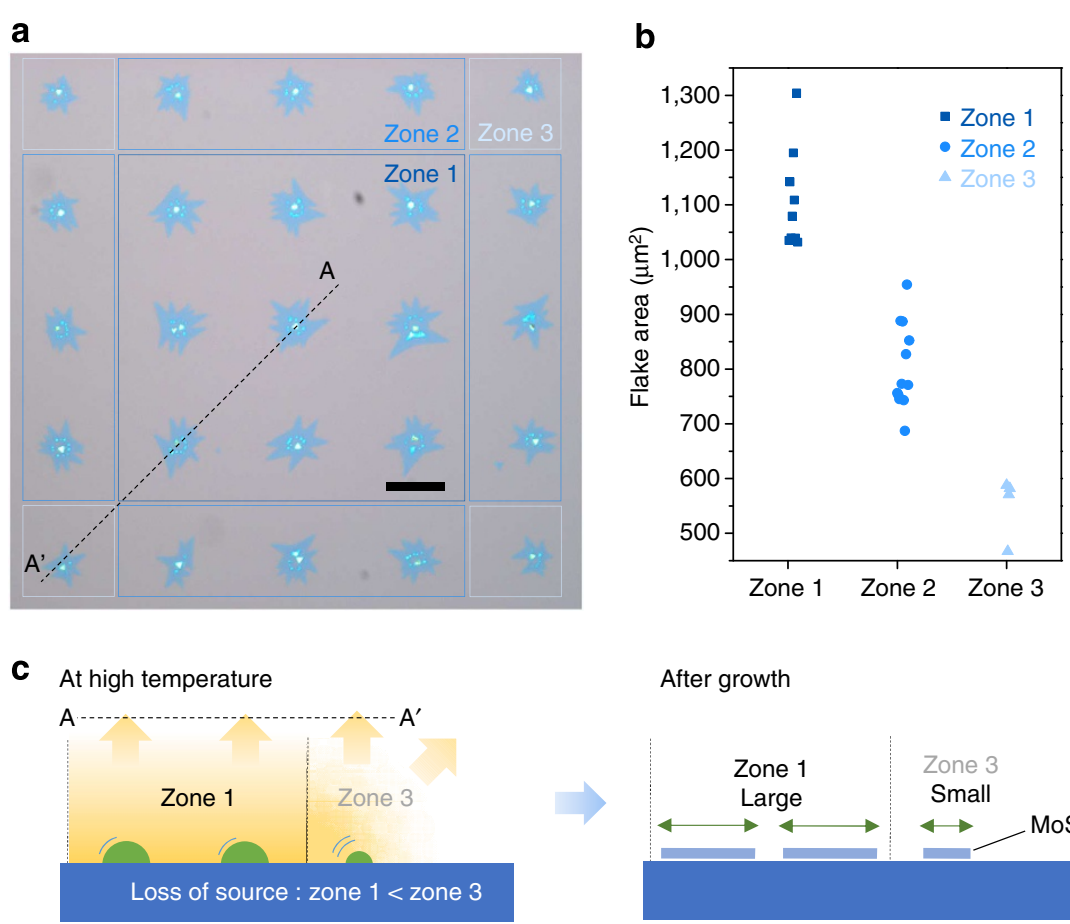

b

After growth

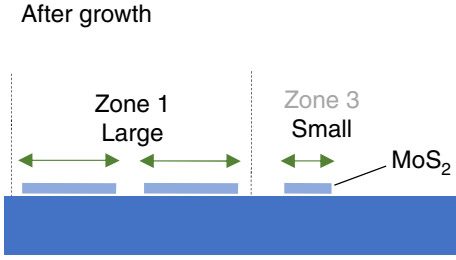

Presumably, these conditions allowed for evaporation of Mo from the growth seed, which then reacted with the injected sulphur to form $\mathrm{MoS}_{2}$ crystallites in regions between the seeds.

Structural and optical property measurements. To demonstrate the versatility of the growth technique, we synthesized a set of monolayer $\mathrm{MoS}_{2}$ flakes to spell out the acronym for the Nano-Bio Interface Center at the University of Pennsylvania, 'NBIC@ UPENN' (Fig. 3a). The centre-to-centre spacing between adjacent seeds was ca $15 \mu \mathrm{m}$. Atomic force microscopy (AFM) was used to confirm that the height of a typical flake was $0.7 \mathrm{~nm}$ (Fig. 3b, inset; additional line scan data are provided in Supplementary Fig. 6. AFM images of other representative monolayer flakes of $\mathrm{MoS}_{2}$ are provided in Supplementary Fig. 7), as expected for monolayer $\mathrm{MoS}_{2}$ (refs 1,13,15,25,26). The Raman spectrum of the monolayer region of the flakes (Fig. $3 \mathrm{c}$ ) showed the expected peak spacing of $19 \mathrm{~cm}^{-1}$ between the $E_{2 g}$ and $A_{1 g}$ modes. Raman mapping (Fig. 3e,f) was used to confirm that the Raman spectrum was uniform over the monolayer region as well as distinct from the spectra associated with multilayer regions and the substrate ${ }^{25-27}$ (Maps of Raman peak positions are also shown in Supplementary Fig 8.). We performed PL and reflection measurements to confirm that the existence of a direct bandgap, which is known to be characteristic of monolayer $\mathrm{MoS}_{2}$ (refs 28-31). Peak features associated with the A $(1.83 \mathrm{eV}$ in $\mathrm{PL}$ and $1.91 \mathrm{eV}$ in reflection measurement) and $\mathrm{B}(1.97 \mathrm{eV}$ in $\mathrm{PL}$ and $2.07 \mathrm{eV}$ in reflection measurement) excitons were also observed (Fig. 3f,g), confirming that the $\mathrm{MoS}_{2}$ grown by the seeded CVD process shows the same characteristics as monolayer material fabricated by mechanical exfoliation ${ }^{32}$.

Sample crystallinity was further characterized with bright-field imaging and selected area electron diffraction (SAED) measurements conducted in the transmission electron microscope (TEM).

Figure 2 | Growth of molybdenum disulphide. (a) $5 \times 5$ array of flakes of monolayer $\mathrm{MoS}_{2}$ grown by chemical vapour deposition. Scale bar is $50 \mu \mathrm{m}$. Different growth zones, with different average size of the flakes (see b), are indicated by outlining: dark blue (zone 1), blue (zone 2) and light blue (zone 3). (b) Areas of individual flakes for zones 1, 2 and 3 in a. Large $\left(500-1300 \mu \mathrm{m}^{2}\right.$ ) flakes of crystalline, monolayer $\mathrm{MoS}_{2}$ (with small multilayer regions) were grown from the seed particles. The size of the flakes is largest in zone 1 and smallest in zone 3. (c) Schematic illustrating the proposed mechanism underlying the observation that the growth process results in larger $\mathrm{MoS}_{2}$ flakes in the interior of the array and smaller flakes towards the edges. 

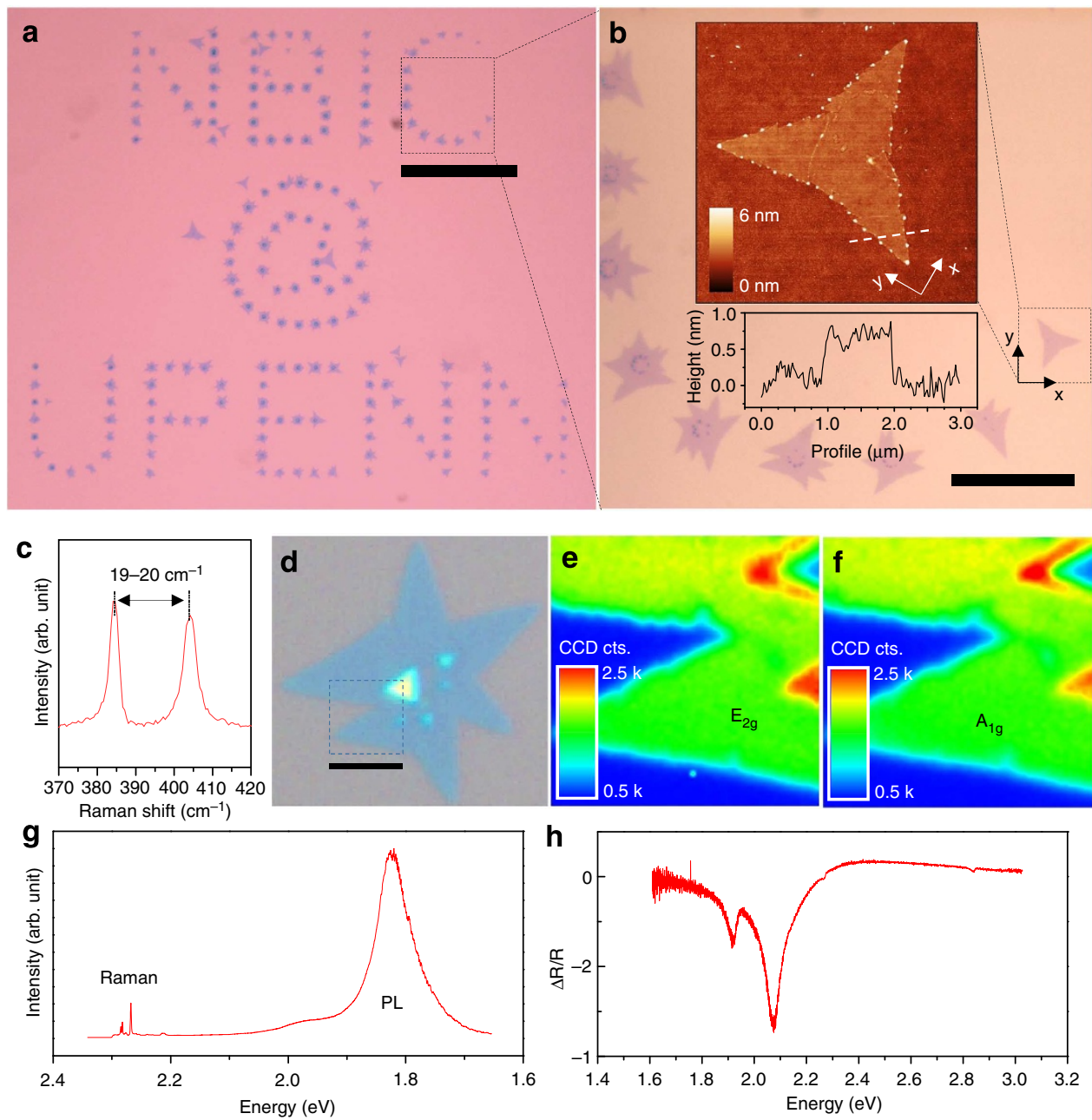

Figure 3 | AFM and optical analysis of $\mathbf{M o S}_{\mathbf{2}}$ flakes. (a) Optical micrograph of $\mathrm{MoS}_{2}$ flakes grown at locations that form NBIC @ UPENN. Scale bar, $100 \mu \mathrm{m}$. (b) Selected area (dashed square in a) at higher magnification. Scale bar, $20 \mu \mathrm{m}$. Inset: AFM image of selected MoS $\mathrm{S}_{2}$ flake (dashed square). Scan image is $10 \times 10 \mu \mathrm{m}^{2}$. (c) Raman signal from the flake shows the $E_{2 g}$ and $A_{1 g}$ peaks with a spacing $\left(19 \mathrm{~cm}^{-1}\right.$ ) characteristic of monolayer MoS 2 . (d) Optical image of $\mathrm{MoS}_{2}$ flake from the array in Fig. 2a. Scale bar, $10 \mu \mathrm{m}$. (e,f) 2D Raman maps taken from the flake shown in $\mathbf{d}$ (dashed line) for the $\mathrm{E}_{2 \mathrm{~g}}$ mode (e) and $\mathrm{A}_{1 \mathrm{~g}}$ mode (f). Scan images are $10 \times 10 \mu \mathrm{m}^{2}$. (g) Photoluminescence $(\mathrm{PL})$ spectrum for the flake shows strong emission associated with the A and B excition at $1.83 \mathrm{eV}$ and $1.97 \mathrm{eV}$, respectively. Strong $\mathrm{PL}$ is characteristic of monolayer $\mathrm{MoS}_{2}$ and is absent in multilayer films. (h) Differential reflectance spectrum showing the $A$ and $B$ exciton features.

A gold grid was first patterned onto a $\mathrm{MoS}_{2}$ flake to prevent tearing of the flake when it was transferred onto a TEM grid covered by a carbon membrane with $1-\mu \mathrm{m}$ diameter holes (Fig. $4 \mathrm{a}$ and Supplementary Fig. 9). To minimize damage to the sample, imaging and SAED were conducted using an accelerating voltage of $80 \mathrm{kV}$. The TEM bright-field images are shown in Fig. 4a,b. Triangular shaped multilayer $\mathrm{MoS}_{2}$ flakes were observed very close to the seed particle, possibly due to limited diffusion of the source during the growth time. Further from the seed, flower-petal-like monolayer $\mathrm{MoS}_{2}$ regions were observed (dashed square in Fig. 4a,b; see Supplementary Fig. 10 for the raw image used to create Fig. $4 \mathrm{~b}$ and an atomic resolution image of a monolayer $\mathrm{MoS}_{2}$ flake). The crystalline structure of the $\mathrm{MoS}_{2}$ was investigated by SAED. Using the intensities of the first- and/or second-order diffraction spots ${ }^{33,34}$, the flower-petallike structures were identified as monolayer $\mathrm{MoS}_{2}$ (see Supplementary Fig. 11). Individual single-crystal $\mathrm{MoS}_{2}$ domains, labelled $\mathrm{A}-\mathrm{D}$ in Fig. $4 \mathrm{~b}$, were distinguished by the orientations of the diffraction spots in SAED (Fig. 4c-f). The data indicate that each domain is well crystallized and their orientations are uncorrelated.
Fabrication and measurement of electronic devices. An etch step is typically used to define individual devices from a continuous layer of material, which creates the risk of degrading a two-dimensional material as well as added processing cost. In contrast, the patterned growth approach to monolayer $\mathrm{MoS}_{2}$ outlined here has the advantage of enabling direct fabrication of multiple devices (Fig $5 \mathrm{a}-\mathrm{f}$ ), without the need for etching. To demonstrate process compatibility with conventional manufacturing, an array of $\mathrm{MoO}_{3}$ growth seeds was patterned by e-beam lithography, and $\mathrm{MoS}_{2}$ monolayer flakes were synthesized as described above. As the $\mathrm{MoS}_{2}$ flakes were grown at known, pre-patterned locations, it would be straightforward to align an electrode pattern to the $\mathrm{MoS}_{2}$ flakes, with no need for a separate etch step. After fabrication of the electrodes, the sample was annealed at $150^{\circ} \mathrm{C}$ at $10^{-5} \mathrm{Torr}$ for $1 \mathrm{~h}$. Figure $5 \mathrm{~g}$ shows the $I_{\mathrm{ds}}-V_{\mathrm{g}}$ characteristics for a set of devices prepared using this approach, measured at a pressure of $40 \mathrm{mTorr}$. Device mobilities were between 8.2 and $11.4 \mathrm{~cm}^{2} \mathrm{~V}^{-1} \mathrm{~s}^{-1}$ and the on/off ratios were in excess $10^{6}$ comparable to earlier best reports for exfoliated $\mathrm{MoS}_{2}$ monolayers ${ }^{14,15,32,33}$. 


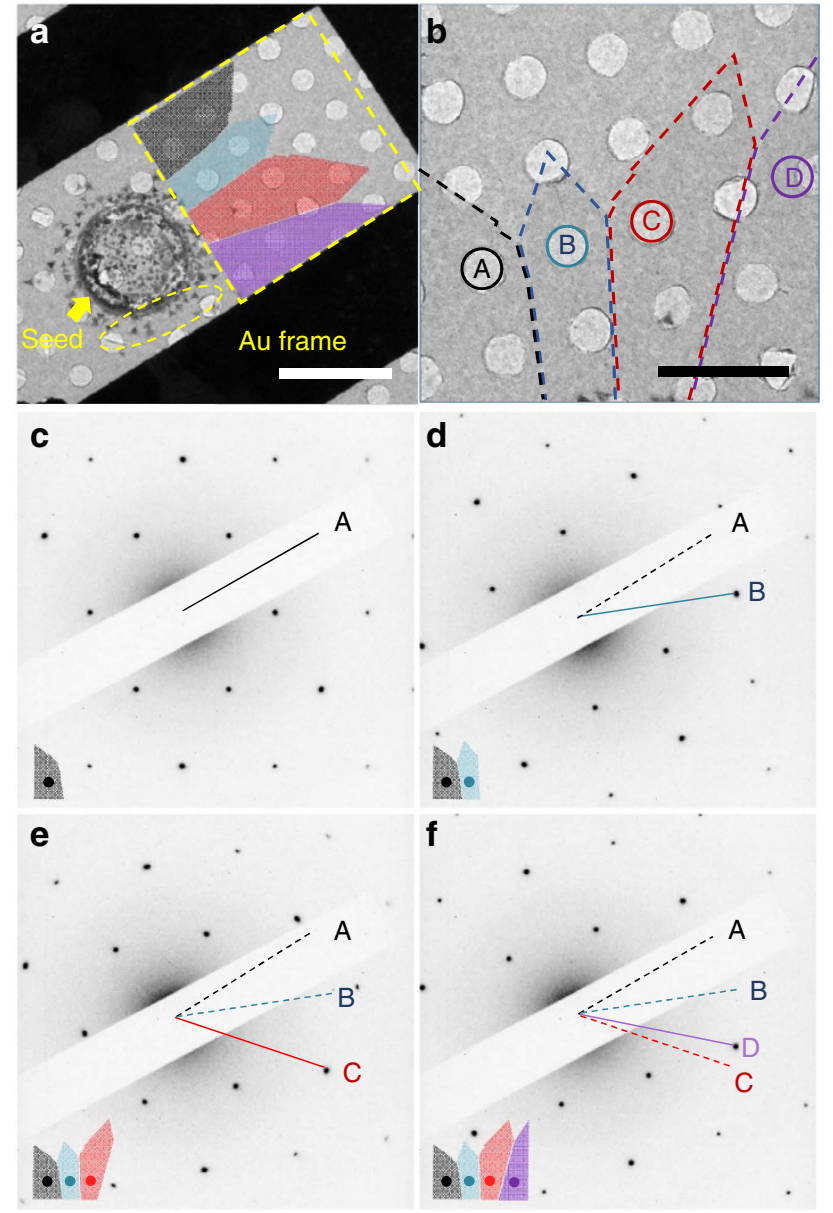

Figure 4 | Transmission electron micrographs and electron diffraction data. (a) Monolayer $\mathrm{MoS}_{2}$ regions are coloured grey, light blue, red and purple (top-bottom) according to their crystallographic orientation. The black regions are parts of a gold frame used to prevent tearing during the transfer process (see main text and Supplementary Fig. 6). Triangular shaped multilayer $\mathrm{MoS}_{2}$ flakes were formed in the seed region, indicated by the yellow arrow. (b) Bright-field (BF) image from the square region outlined by a dashed white line in $\mathbf{a}$. Scale bars in fig $\mathbf{a}$ and $\mathbf{b}$ are $5 \mu \mathrm{m}$ and $3 \mu \mathrm{m}$, respectively. (c-f) Electron diffraction data from the regions labelled $A-D$ in $\mathbf{b}$.

\section{Discussion}

In summary, we have developed a novel patterned growth method for flakes of highly crystalline monolayer $\mathrm{MoS}_{2}$, where molybdenum-containing growth seeds (AHM or $\mathrm{MoO}_{3}$ ) are defined using conventional lithographic methods. This technique should be generalizable to the growth of any layered transition metal dichalcogenide, and it creates a pathway to controllably tailor the size and position of the material. Control of growth parameters enables synthesis of high quality, crystalline, monolayer $\mathrm{MoS}_{2}$ with electrical and optical properties comparable to exfoliated material. The process enables straightforward fabrication of integrated circuits without the need for a separate etch step. The work represents an important advance towards scalable production of high-quality layered transition metal dichalcogenide-based devices and circuits. An ongoing challenge to the field is patterned growth of monolayer, single-crystalline domain $\mathrm{MoS}_{2}$ at predefined locations.

\section{Methods}

Preparation of patterned molybdenum sources. Electron beam or photolithography was used to prepare $5 \times 5$ arrays of $5-\mu \mathrm{m}$ square windows. A $7 \times 7$ array of 3- $\mu \mathrm{m}$ square windows was used for the experiment that resulted in the growth of exclusively monolayer $\mathrm{MoS}_{2}$ flakes (see Supplementary Fig. 4). Molybdenum-containing source material was then deposited by either spin coating ( 1,000 r.p.m. for 30 s, followed by 3,000 r.p.m. for $10 \mathrm{~s}$ ) with a saturated solution of AHM (product number A674, Fisher Scientific) in deionized water or thermal evaporation of 150 -nm-thick molybdenum trioxide $\left(\mathrm{MoO}_{3}\right.$, product number 203815, Sigma-Aldrich). The resist was removed using acetone followed by isopropyl alcohol, and the sample was then blown dry with compressed nitrogen gas. An aggregation step was then performed to obtain regular beads of the source material. Although monitoring the sample under an optical microscope, the substrate was cooled until the hygroscopic $\mathrm{AHM}$ or $\mathrm{MoO}_{3}$ absorbed water, forming droplets at the seed locations. As the temperature was increased back to room temperature, the water evaporated, leaving behind rounded, uniform, beads of AHM or $\mathrm{MoO}_{3}$ as shown in Supplementary Fig. 2. Samples were then annealed at $300{ }^{\circ} \mathrm{C}$ for $20 \mathrm{~min}$ in a flow of 500 s.c.c.m. of nitrogen gas ( $99.999 \%$ purity). To promote $\mathrm{MoS}_{2}$ growth, the sample was treated with oxygen plasma $(65 \mathrm{~W}$, $300 \mathrm{mTorr}, 2 \mathrm{~min}$ ) and then spin-coated (3,000 r.p.m., $1 \mathrm{~min}$ ) with a surfactant solution $(0.1-1 \%$ solution of sodium cholate in deionized water).

Growth process. Samples with patterned growth seeds were placed into a 1-inch CVD furnace with a 25-mg solid sulphur source (part number 213292, SigmaAldrich) at the upstream end. The furnace temperature was increased to $750^{\circ} \mathrm{C}$ at a ramp rate of $70^{\circ} \mathrm{C} \mathrm{min}^{-1}$ under a nitrogen (99.999\%) flow of 700 s.c.c.m. The temperature was held constant for 20 min during the $\mathrm{MoS}_{2}$ growth phase, and the system was then rapidly cooled to room temperature. It was noted that the sulphur source started to melt when the temperature in the middle of the furnace was ca $650^{\circ} \mathrm{C}$.
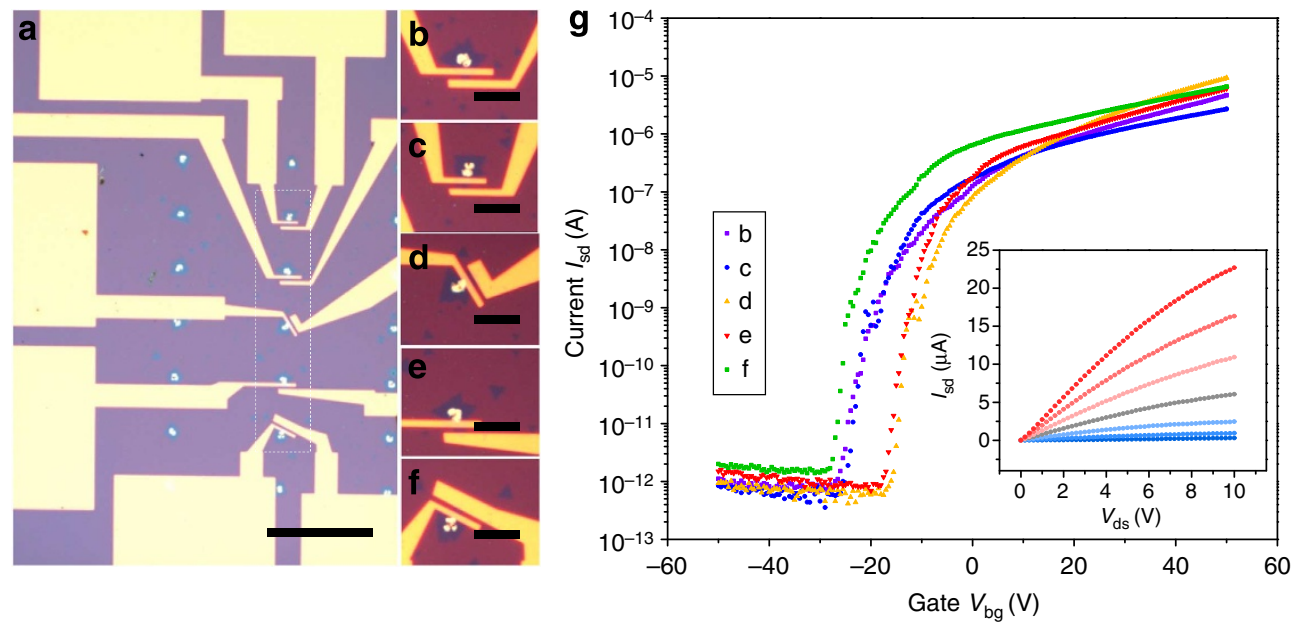

Figure 5 | Device fabrication and $\mathbf{I}-\boldsymbol{V}$ measurement. (a) Optical micrograph of devices that was fabricated from $\mathrm{MoS}_{2}$ grown by the patterned seed technique. Scale bar, $100 \mu \mathrm{m}$. (b-f) Higher magnification images of the rectangular region indicated in $\mathbf{a}$. Scale bar, $20 \mu \mathrm{m}$. (g) $I_{\mathrm{ds}}-V_{\mathrm{g}}$ characteristics for the devices in b-f with $1 \mathrm{~V} V_{\text {ds }}$. Inset: $I_{\mathrm{ds}}-V_{\mathrm{ds}}$ characteristics at several back gate voltages. 
Device fabrication and current-voltage measurements. After growth of monolayer $\mathrm{MoS}_{2}$ flakes from patterned growth seeds, electron beam lithography was used to define an electrode pattern that was aligned to the growth sites. The electrode metallization consisted of $1 \mathrm{~nm} \mathrm{Cr}$ and $30 \mathrm{~nm}$ Au deposited by e-beam evaporation. After metallization, devices were annealed for $60 \mathrm{~min}$ at $150^{\circ} \mathrm{C}$ at a pressure of $10^{-5}$ Torr. Current-voltage measurements were conducted at room temperature under a vacuum of $40 \mathrm{mTorr}$

\section{References}

1. Radisavljevic, B., Radenovic, A., Brivio, J., Giacometti, V. \& Kis, A. Single-layer $\mathrm{MoS}_{2}$ transistors. Nat. Nanotechnol. 6, 147-150 (2011).

2. Jariwala, D. et al. Gate-tunable carbon nanotube- $\mathrm{MoS}_{2}$ heterojunction $p-n$ diode. Proc. Natl Acad. Sci. USA 110, 18076-18080 (2013).

3. Zhang, W. et al. High-gain phototransistors based on a CVD $\mathrm{MoS}_{2}$ monolayer. Adv. Mater. 25, 3456-3461 (2013).

4. Bao, W. Z., Cai, X. H., Kim, D., Sridhara, K. \& Fuhrer, M. S. High mobility ambipolar $\mathrm{MoS}_{2}$ field-effect transistors: Substrate and dielectric effects. Appl. Phys. Lett. 102, 042104 (2013)

5. Jariwala, D., Sangwan, V. K., Lauhon, L. J., Marks, T. J. \& Hersam, M. C. Emerging device applications for semiconducting two-dimensional transition metal dichalcogenides. ACS Nano 8, 1102-1120 (2014).

6. Yu, W. J. et al. Vertically stacked multi-heterostructures of layered materials for logic transistors and complementary inverters. Nat. Mater. 12, 246-252 (2013).

7. Yu, W. J. et al. Highly efficient gate-tunable photocurrent generation in vertical heterostructures of layered materials. Nat. Nanotechnol. 8, 952-958 (2013).

8. Chang, H. Y. et al. High-performance, highly bendable $\mathrm{MoS}_{2}$ transistors with high-k dielectrics for flexible low-power systems. ACS Nano 7, 5446-5452 (2013).

9. Salvatore, G. A. et al. Fabrication and transfer of flexible few-layers $\mathrm{MoS}_{2}$ thin film transistors to any arbitrary substrate. ACS Nano 7, 8809-8815 (2013)

10. Radisavljevic, B., Whitwick, M. B. \& Kis, A. Integrated circuits and logic operations based on single-layer $\mathrm{MoS}_{2}$. ACS Nano 5, 9934-9938 (2011).

11. Zhan, Y., Liu, Z., Najmaei, S., Ajayan, P. M. \& Lou, J. Large-area vapor-phase growth and characterization of $\mathrm{MoS}_{2}$ atomic layers on a $\mathrm{SiO}_{2}$ substrate. Small 8, 966-971 (2012).

12. Liu, K. K. et al. Growth of large-area and highly crystalline $\mathrm{MoS}_{2}$ thin layers on insulating substrates. Nano Lett. 12, 1538-1544 (2012).

13. Lee, Y. H. et al. Synthesis of large-area $\mathrm{MoS}_{2}$ atomic layers with chemical vapor deposition. Adv. Mater. 24, 2320-2325 (2012).

14. van der Zande, A. M. et al. Grains and grain boundaries in highly crystalline monolayer molybdenum disulphide. Nat. Mater. 12, 554-561 (2013).

15. Najmaei, S. et al. Vapour phase growth and grain boundary structure of molybdenum disulphide atomic layers. Nat. Mater. 12, 754-759 (2013).

16. Lee, Y. H. et al. Synthesis and transfer of single-layer transition metal disulfides on diverse surfaces. Nano Lett. 13, 1852-1857 (2013).

17. Ling, X. et al. Role of the seeding promoter in $\mathrm{MoS}_{2}$ growth by chemical vapor deposition. Nano Lett. 14, 464-472 (2014).

18. Nam, H. et al. $\mathrm{MoS}_{2}$ Transistors fabricated via plasma-assisted nanoprinting of few-layer $\mathrm{MoS}_{2}$ flakes into large-area arrays. ACS Nano 7, 5870-5881 (2013).

19. Park, W. et al. Photoelectron spectroscopic imaging and device applications of large-area patternable single-layer $\mathrm{MoS}_{2}$ synthesized by chemical vapor deposition. ACS Nano 8, 4961-4968 (2014).

20. Li, H. et al. Controlled synthesis of topological insulator nanoplate arrays on mica. J. Am. Chem. Soc. 134, 6132-6135 (2012).

21. Yu, Q. et al. Control and characterization of individual grains and grain boundaries in graphene grown by chemical vapour deposition. Nat. Mater. 10, 443-449 (2011).

22. Wei, Wu. et al. Growth of single crystal graphene arrays by locally controlling nucleation on polycrystalline $\mathrm{Cu}$ using chemical vapor deposition. Adv. Mater. 23, 4893-4903 (2011)

23. Hanafi, Z. M., Khilla, M. A. \& Askar, M. H. The thermal decomposition of ammonium heptamolybdate. Thermochim. Acta 45, 221-232 (1981).
24. Wienold, J., Jentoft, R. E. \& Ressler, T. Structural investigation of the thermal decomposition of ammonium heptamolybdate by in situ XAFS and XRD. Eur. J. Inorg. Chem. 2003, 1058-1071 (2003).

25. Lee, C. et al. Anomalous lattice vibrations of single- and few-layer $\mathrm{MoS}_{2}$. ACS Nano 4, 2695-2700 (2010).

26. Li, H. et al. From bulk to monolayer $\mathrm{MoS}_{2}$ : evolution of raman scattering. $A d v$. Funct. Mater. 22, 1385-1390 (2012).

27. Gong, C. et al. Metal contacts on physical vapor deposited monolayer $\mathrm{MoS}_{2}$. ACS Nano 7, 11350-11357 (2013).

28. Mak, K. F., Lee, C., Hone, J., Shan, J. \& Heinz, T. F. Atomically thin $\mathrm{MoS}_{2}$ : a new direct-gap semiconductor. Phys. Rev. Lett. 105, 136805 (2010).

29. Sundaram, R. S. et al. Electroluminescence in single layer $\mathrm{MoS}_{2}$. Nano Lett. 13, 1416-1421 (2013).

30. Splendiani, A. et al. Emerging photoluminescence in monolayer $\mathrm{MoS}_{2}$. Nano Lett. 10, 1271-1275 (2010)

31. Shi, H. et al. Exciton dynamics in suspended monolayer and few-layer $\mathrm{MoS}_{2} 2 \mathrm{D}$ crystals. ACS Nano 7, 1072-1080 (2013).

32. He, K., Poole, C., Mak, K. F. \& Shan, J. Experimental demonstration of continuous electronic structure tuning via strain in atomically thin $\mathrm{MoS}_{2}$. Nano Lett. 13, 2931-2936 (2013).

33. Brivio, J., Alexander, D. T. L. \& Kis, A. Ripples and layers in ultrathin $\mathrm{MoS}_{2}$ membranes. Nano Lett. 11, 5148-5153 (2011).

34. Ping, J. \& Fuhrer, M. S. Layer number and stacking sequence imaging by transmission electron microscopy. Nano Lett. 12, 4635-4641 (2012).

\section{Acknowledgements}

This work was supported by the National Science Foundation Accelerating Innovation in Research Program AIR ENG-1312202 and the Nano/Bio Interface Center NSF NSEC DMR08-32802. B.S.L., J.H.P. and R.A. were supported by the National Institutes of Health through the NIH Director's New Innovator Award Program, 1-DP2-7251-01 and the US Army Research Office under Grant No. W911NF-11-1-0024. R.A. and A.T.C.J. acknowledge Seed Project support from the LRSM, NSF MRSEC DMR-1120901. G.H.H., S.Y.L. and Y.H.L. acknowledge IBS-R011-D1 (Institute for Basic Science). We acknowledge use of facilities associated with the Nano/Bio Interface Center (Raman spectroscopy, AFM) and the Nanoscale Characterization Facility (TEM) at the University of Pennsylvania. The aberration-corrected TEM image presented in Supplementary Fig. 9 was obtained by Zhengqing John Qi and Julio A. Rodríguez-Manzo (Drndic group, University of Pennsylvania), using an aberration-corrected transmission electron microscope at the Center for Functional Nanomaterials, Brookhaven National Laboratory.

\section{Author contributions}

A.T.C.J. directed the research; G.H.H. proposed and designed the experiment; G.H.H. and C.H.N. carried out $\mathrm{MoS}_{2}$ growth. Electrical measurements were performed by N.J.K. C.H.N. and G.H.H., and also by S.Y.L. under supervision of Y.H.L. N.J.K. and G.H.H. performed AFM and Raman characterization; J.P., J.K., and G.H.H. performed sample preparation and characterization for TEM experiments; B.S.L. and J.H.P. (under the supervision of R.A.) carried out photoluminescence and reflection measurements. G.H.H., N.J.K., C.H.N. and A.T.C.J. wrote the manuscript, with input and approval from all the authors.

\section{Additional information}

Supplementary Information accompanies this paper at http://www.nature.com/ naturecommunications

Competing financial interests: The authors declare no competing financial interests.

Reprints and permission information is available online at http://npg.nature.com/ reprintsandpermissions/

How to cite this article: Han, G. H. et al. Seeded growth of highly crystalline molybdenum disulphide monolayers at controlled locations. Nat. Commun. 6:6128 doi: $10.1038 /$ ncomms7128 (2015). 